\title{
Visualizing Financial Stock Data within an Augmented Reality Trading Environment
}

\author{
Dariusz Rumiński, Mikołaj Maik and Krzysztof Walczak \\ Poznań University of Economics and Business, Department of Information \\ Technology, Niepodległości 10, 61-875 Poznań, Poland \\ [ruminski, maik, walczak]@kti.ue.poznan.pl
}

\begin{abstract}
In this paper, we present a novel augmented reality $(A R)$ system for visualization of financial stock data. The system enhances the equity traders' working environment with virtual charts and live business TV streams, overlaid onto the real trading workspace. The presented research investigates how human cognitive capabilities can be extended by the use of artificial computer-generated $3 D$ representation of financial data and interaction with such data representation using hand gestures. We combined the nVisor ST50 headset with InteriaCube4 and Leap Motion devices to enable tracking of head orientation and controlling the AR environment with hands. With the use of hand gestures, a user is able to create virtual charts displaying real-time stock data and financial TV streams within his/her surroundings. To evaluate the system, we conducted experimental tests with 22 users. Our objective was to evaluate tasks completion times and users' experience while creating and parametrizing virtual stock charts through the AR interface. The obtained results are promising and demonstrate that most users were able to finish tasks in acceptable time without significant difficulties.
\end{abstract}

\section{Introduction}

Professional stock traders are responsible for buying and selling tradable financial assets such as stocks, bonds, futures, options, and swaps - to name a few. They also conduct intensive and extensive research and observation of how financial markets perform, e.g., when new macroeconomic data or other relevant news are published. Traders, in their daily work, use traditional workspaces that may be composed of multiple monitors on which diverse financial information, charts, tables, and indicators are presented simultaneously, as presented in Figure 1a. Moreover, to operate a stock trading system, a standard keyboard and mouse are typically used. Also, a landline is a standard tool of traders to verbally communicate with managers, colleagues, or clients when there is a need to consult and make a buy/sell decision. Moreover, traders often simultaneously track live business TV channels or social media to 'catch' profitable news. In such an environment, a trader has to be focused all the time, while observing continuous 
changes in financial markets. It is easy to make a mistake when, e.g., some economic data are overlooked, and consequently, the trader might produce a loss or miss a potential profit.

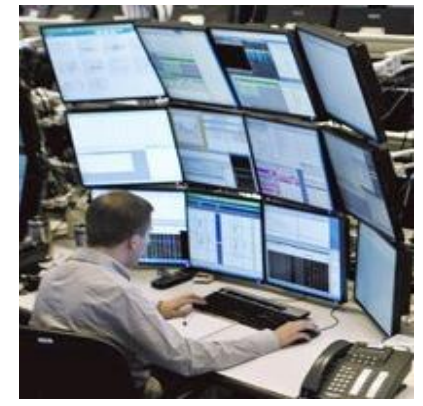

(a)

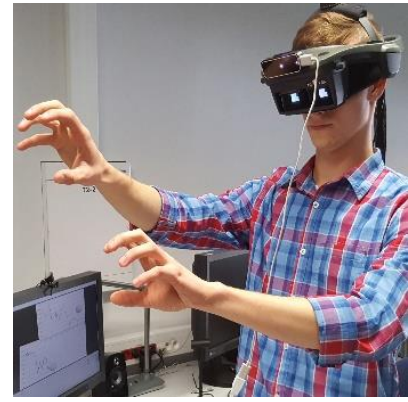

(b)

Figure 1

Fig. (a) Traditional trading workspace with multiple screens; Fig. (b) AR trading workspace controlled with hand gestures.

Given the high amount of information that a trader must simultaneously follow, the high cognitive load and the direct financial impact of their decisions, it is important to study how the cognitive abilities of traders can be extended with the use of modern technology. These kinds of studies fall into the domain of cognitive infocommunication (CogInfoCom) [25-29]. The primary goal of CogInfoCom is to study how cognitive processes can co-evolve with infocommunications devices so that the capabilities of the human brain may not only be extended through these devices, irrespective of geographical distance, but may also interact with the capabilities of any artificially cognitive system [26, 29].

The use of Augmented Reality (AR) technology may aid traders' work, by enabling creation of customized information spaces, in which relevant data are overlaid on the users' real trading workspace in the form of virtual screens supplementing physical screens or even completely replacing such complex computer setups with an AR interface. In the AR system, users can augment their surroundings with virtual charts, tables, indicators, financial TV channels as many as a user needs to observe - without running into hardware- or space- limitation issues. Additionally, perception of handling changes in financial markets can be increased with the use of spatial sound in $\operatorname{AR}[12,13,19]$, and as a result, the trader could perform better, by getting aural notifications triggered within his/her surrounding, e.g., when a particular financial value rapidly changes. Moreover, AR opens new possibilities to remote collaboration within the financial domain, e.g., when there is a need to coach or supervise the trading performance of employees [30]. Previous studies have demonstrated that remote helpers have allowed local workers to perform better real-world tasks with success [1,5]. Also, $3 \mathrm{D}$ visualization of abstract data has been shown useful in previous research work $[2,16]$. 
The presented AR trading system can be operated by hand gestures to manipulate information components in the user's environment. The introduction of motion gestures enables further enhancement of users' performance while interacting with virtual objects. Nowadays, natural user interfaces play an essential role in humancomputer interaction by supporting - or even in some cases completely replacing - traditional computer input devices such as mouse, keyboard, or touchpad to perform operations more precisely and faster [20]. With the introduction of the Microsoft Kinect in 2011 and the notable Leap Motion in 2015, technology for natural interaction is available at a price affordable to many consumers as well as researchers. Hand pose estimation is now achievable without the need for data gloves or external sensing [17]. This allows for designing more complex natural hand interaction within AR and VR applications than could be achieved in past research $[4,6,8,9,14]$.

Despite the increasing popularity of AR interfaces, there is still a lack of interaction techniques that allow to fully benefit from the above-mentioned technologies - especially in financial markets. Therefore, it is important to study natural hand interaction supporting $\mathrm{AR}$ systems to learn its limitations and possibilities within business domains.

In the remainder of this paper, first an overview of the related work is provided, then the technical setup is presented, followed by a description of the developed system. Next, user evaluation tests are presented together with results obtained from the experimental study. Finally, conclusions are provided and directions for future research are discussed.

\section{Related Works}

Augmented reality (AR) is a field of computer science that concerns computer vision-based technologies enabling superimposing rich computer-generated content - such as 2D and 3D multimedia objects - in real time, on a view of real objects. Although the term of AR became popular in the scientific community only after the publication of a special issue of the ACM Communication journal in 1993 devoted to this subject [21], the first AR display described in the literature dates back to 1968 [22]. Milgram et al. differentiated AR from VR using the Reality-Virtuality continuum concept [23]. In turn, Azuma defined AR as any AR system that meets the following requirements: it combines real and virtual; is interactive in real time; and is registered in 3D [24]. AR has a key role in the field of cognitive infocommunication (CogInfoCom), as defined by Baranyi and Csapó, by extending human surroundings with new synthetic forms of information presentation and enabling rich forms of interaction with such mixed reality environments [25]. 
Several studies that have had a significant impact on AR, as well as, VR development concerned the possibilities of using hands for user interaction. For instance, Piumsomboon et al. provided a user-defined gesture set that can be used in AR systems and conducted an experimental study of guess-ability for hand gestures in $\mathrm{AR}$, in which 800 gestures were elicited for 40 selected tasks from 20 participants [10]. Researchers found that most of the gestures obtained from the participants were physical gestures (39\%) for tasks such as move, rotate, scale, and delete.

The knowledge from the previous study has been used to improve the understanding of natural hand interaction and ultimately create novel natural hand interaction techniques that enhance user experience when interacting with $3 \mathrm{D}$ computer-generated content [11]. To better understand natural hand interaction, researchers developed AR interaction framework, GSIAR, which provides two interaction techniques - so-called G-Shell and G-Speech. Researchers demonstrated that direct free-hand interaction techniques can be natural, intuitive and precise. Additionally, the use of the G-Speech method, allowed to observe that ease of use and control is achievable for interactions without direct contact. Authors recommend combining both interaction techniques in a single AR interface to improve usability and enhance the user experience.

The accuracy and precision of hand gestures have been tested with the use of the Leap Motion controller by Valentini and Pezzuti [15]. Authors conducted an experimental study of assessing the accuracy of the Leap Motion Controller in tracking of fingertips. The assessment was performed in a real context using volunteers who were asked to point with fingers to a specific location in space. Results show that Leap Motion is suitable for robust tracking of the user's hands. The results also unveil that there are preferable zones in which the tracking performance is better.

Chang et al. compared two annotation methods using the HoloLens device - AirDrawing and Surface-Drawing - with either raw but smoothed or interpreted and beautified gesture input [3]. The methods have different characteristics regarding allowed cursor control. Authors performed an experiment in which users used the above-mentioned methods to draw on two separate real-world objects at various distances. In the Surface-Drawing mode, users control a cursor that is projected onto the world model, allowing gesture input to occur directly on surfaces of realworld objects. In the second method, called Air-Drawing, gesture drawing occurs at the user's fingertip and is projected onto the world model on release. Results indicated that Surface-Drawing is more accurate than Air-Drawing.

The next research [18] that provides valuable cues while designing an AR system for traders presents the use of novel 3D virtual reality keyboard system with realistic haptic feedback. The presented method uses two five-fingered hand data to track finger positions and postures. Moreover, it uses micro-speakers to create vibrations, while an HMD is used to display virtual hands and keyboard. The 
results of this study show the advantages of the haptic VR keyboard - the keyboard that can pop-up at any location in a VR environment and also can be used to provide realistic key-click haptic feedback - over a physical keyboard. While the solution has been implemented for VR, it could be easily adapted to AR taking into account the lesson learned.

The next article [7] that influenced our research explores the effects of adding gesture interaction with 3D synthetic content. The system in the study comprises a Leap Motion sensor to track the user's hand in combination with a SoftKinetic RGB-D camera to capture the texture of hands. It implements two different handvisualization modes for $360^{\circ}$ movie watching experience: point-cloud of the real hand and a rigged computer-generated hand. The results of the research demonstrate that participants prefer the conditions with realistic hand representation, and they feel stronger embodiment and ownership. These results suggest that the interaction with virtual objects should be performed using a real hand visualization instead of the virtual hand model.

\section{Technical Setup}

In order to develop the prototype of the AR Trading system, we needed a highresolution HMD device. We used nVisor ST50 (Fig. 2a) that is built with the use of high-contrast OLED micro-displays. The HMD provides 1280 by 1024-pixel resolution per eye in a low-power, 50-degree field of view compact design, making the see-through compatible optics suitable for professional AR applications. The nVisor ST50 device supports the use of standard motion tracking devices from InterSense, Ascension, Polhemus, and others via a tracker platform mounted on the back of the HMD.

To detect the movement and position of the user's head, we attached the InteriaCube 4 device at the back of the nVisor ST50 HMD (Fig. 2c). The InertiaCube4 sensor integrates the latest in MEMS inertial technology and utilizes advanced Kalman filtering algorithms to produce a full 360 degree orientation tracking. The sensor is compact and portable. The device is suitable for a wide range of applications including simulation, training, virtual and augmented reality, motion capture, and human movement analysis. 


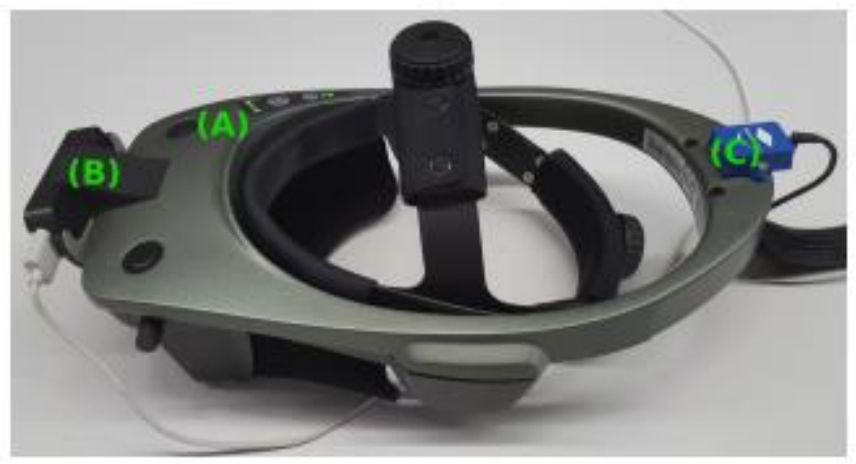

Figure 2

The prototype AR Trading system hardware consisting of the nVisor ST50 HMD (a), with attached Leap Motion (b) and InteriaCube4 (c) sensors

In order to control AR Trading visual components with the use of hand gestures we used a Leap Motion controller and attached it to the front of the HMD (Fig. 2b). The Leap Motion controller is a small USB device designed for use on a virtual reality headset or on a computer desk. It uses two monochromatic IR cameras and three infrared LEDs. The device observes a roughly hemispherical area, to a distance of about 1 meter. The LEDs generate pattern-less IR light, and the cameras capture almost 200 frames per second of reflected light data. These data are then sent through a USB cable to the host computer, where they are analyzed by the Orion software.

\section{System Software}

The software part of the AR Trading system consists of the MiddleVR and the Orion libraries together with a custom application designed in Unity 3D. MiddleVR is a library responsible for handling input devices, stereoscopy, and clustering. It allows to quickly integrate hardware components listed in Section 3. Moreover, it offers an easy-to-use graphical user interface to configure VR/AR applications. In order to handle hand tracking in the $3 \mathrm{D}$ space, we use the Orion software. All components have been combined within cross-platform game engine Unity 3D (5.6.0f3 64-bit version). The AR Trading application has been implemented within Unity $3 \mathrm{D}$ with the use of the C\# language. The financial component providing stocks data is based on Google Finance API.

When the AR Trading application starts, a menu that informs a user about the possible modes of operation appears. The system supports three modes of operation, which can be activated with the use of three buttons in the menu. By pressing the button on the right, a user can go into the presentation mode (Mode 1), which is designed to quickly show the program capabilities without engaging 
advanced interactions. Several predefined charts are displayed, which can be viewed by the user (as presented in Fig. 3).

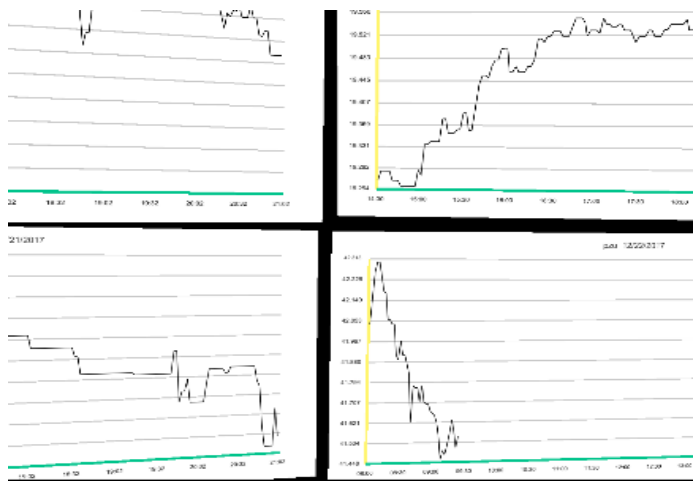

Figure 3

Mode 1 - demonstrating predefined virtual charts

This mode has been developed to demonstrate the system in a quick way without the need of creating and configuring new charts. All gesture functions are turned off; it is only possible to return to the main menu by holding the left thumb down for 3 seconds. We have added two virtual spheres at the palm of each hand, which indicate whether the Leap Motion controller is working correctly. When the user's hands are close to a virtual object, the spheres change color to blue, signaling the user about the possibility of grabbing a virtual object.

A button in the middle of the main menu takes a user to the Mode 2 - a tutorial in which the user is able to learn the basic steps of creating and parametrizing virtual charts. In this mode, the user can practice hand gestures responsible for grabbing and moving cubes and ready-made virtual charts. The user can also train typing on a virtual keyboard to fill a blank chart with data. All user's activities are logged and completion times of particular tasks are measured.

At the beginning of the tutorial mode (Mode 2), a user sees a visual instruction informing about the first task - exchanging positions of virtual cubes. The goal of this tasks is to train the pinching gesture. After the information is displayed, the user is asked to press a button that starts the Task \#1. After that, two objects appear in front of the user - red and blue cubes - positioned below the left user's hand. The user has to exchange their positions by putting the red cube in the frame of a blue cube and vice versa. Each task is finished by pressing a button that appears on the right side of the user. Figure 4 depicts a user practicing the pinching gesture.

After completing Task \#1, an instruction is displayed informing how to type correctly on the virtual keyboard in Task \#2. A user presses the keyboard buttons with the palms of his/her hands or by hitting them with fists. 

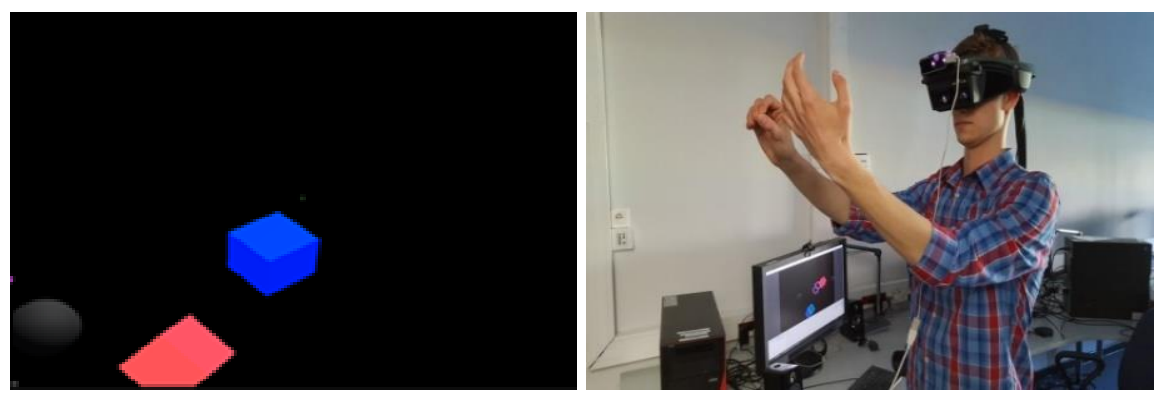

Figure 4

Practicing Task \#1 - changing positions of virtual cubes

To avoid problems with pressing several buttons at the same time, finger collision with keyboard buttons has been disabled. We divided the task of typing on the virtual keyboard into two parts. During the first part, the user exercises precision of hitting virtual keys while writing his or her name. During the second part, the user is asked to type the phrase "Badanie.Naukowe" (English translation "Scientific.Research"). The time of the second part is measured. Figure 5 presents a user performing Task \#2.
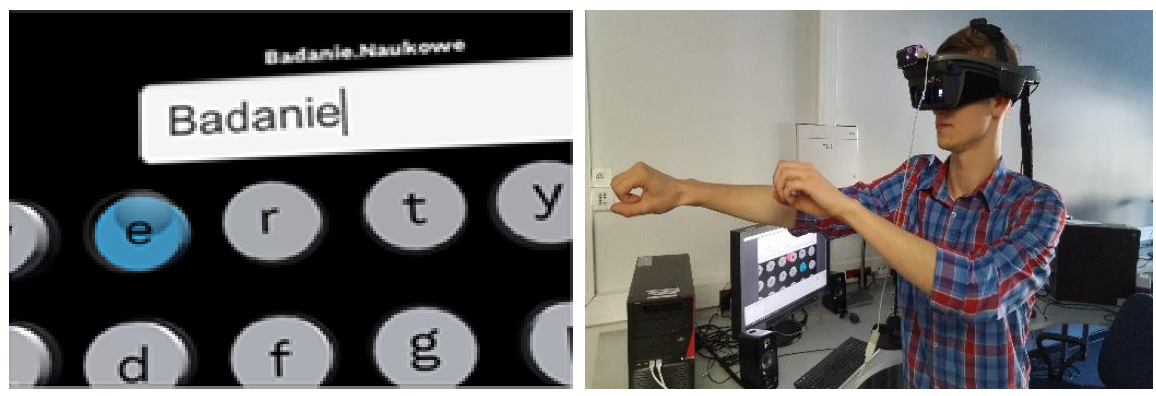

Figure 5

Practicing Task \#2 - writing on a virtual keyboard

Next, Task \#3 starts. Two previously prepared virtual charts are displayed in front of the user - one on the left and another on the right side. The user is asked to grab both and exchange their positions - right chart must be placed in a frame surrounding left chart and vice versa. The distance between the charts has been set in such a way that it is difficult to reach the left chart with the right hand and the right chart with the left hand. Thus, naturally, it forces the user to grab the virtual object positioned on the left side with the use of the left hand and the second object with the use of the right hand. Completion time of this task is also measured. Figure 6 depicts a user performing Task \#3. When a user completes the tutorial, the program returns to the main menu. 

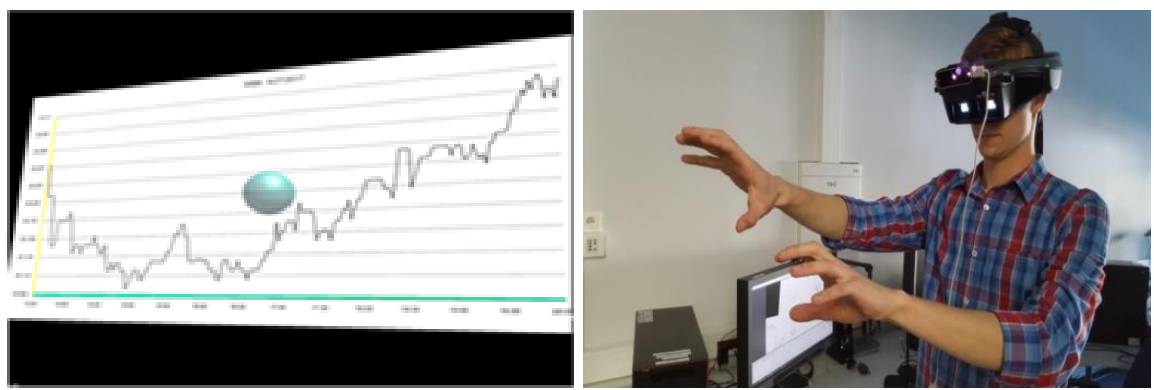

Figure 6

Practicing Task \#3 - exchanging position of virtual charts

When a user presses the button displayed on the left side in the main menu, Mode \#3 of the AR Trading application is activated. Within Mode \#3 - which is the main part of the application - a user can personalize their virtual trading workspace by creating and configuring virtual charts and video-based components used for displaying live TV streams with relevant financial news.

As the first step of forming the personalized workspace, a user needs to open left hand turned in the user's direction. This gesture triggers an interface with two cubes that stick to the user's hand (as presented in Figure 7). The red cube builds an empty virtual chart, while the blue - a financial channel screen. Figure 7 depicts the user interface aligned to the left hand. With the use of MiddleVR GUI, the Leap Motion's hand model was calibrated so that it is aligned as close as possible to the real hand of the user (as presented in Figure 7a). Next, to give the best AR experience, the virtual hand model has been removed (as depicted in Figure $7 b)$.

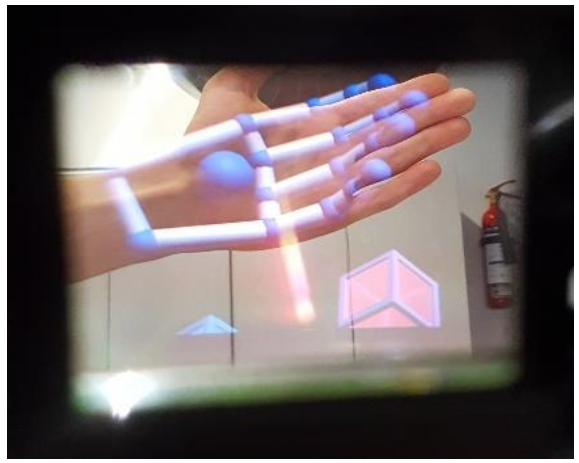

(a)

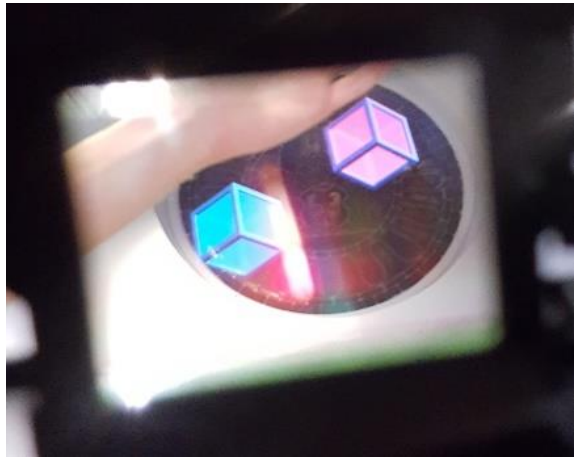

(b)

Figure 7

Interacting with the hand interface - (a) virtual hand model aligned to the user's hand;

(b) interface with removed virtual hand model to give the best AR experience. Pictures taken through the HMD 
Next, a user needs to pinch the red or the blue cube with the right hand and drag it into any place in the surrounding AR environment. After selecting the red object, a blank virtual chart appears. On the left side of the virtual chart, a button responsible for creating a virtual keyboard under the graph appears, as presented in Figure 8a. When the user presses it, a virtual keyboard pops up. The virtual keyboard contains all the letters of the alphabet, shift, backspace, and dot buttons. With the use of the keyboard, a user can enter the ISIN (International Securities Identification Number) of the company whose shares he/she wants to follow (as depicted in Figure 8b). Moreover, the user can switch between stock exchanges and companies by pushing an appropriate input field with their index finger. Just like in the tutorial, text can be typed by pressing the virtual keys with the use of palms or by punching them with fists. The button on the right side of the chart accepts the text the user has typed (Figure 8c). If there are no typos, stock data of selected company are downloaded and displayed. At any time, a user can activate the virtual keyboard again to correct typos or enter the name of another company.

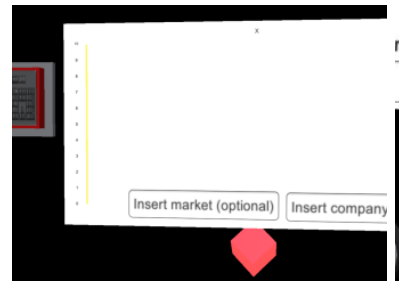

(a)

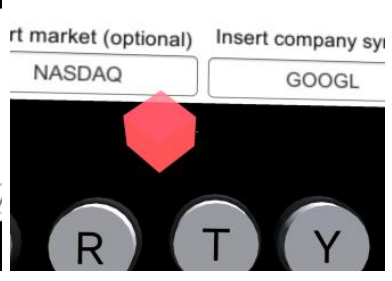

(b)

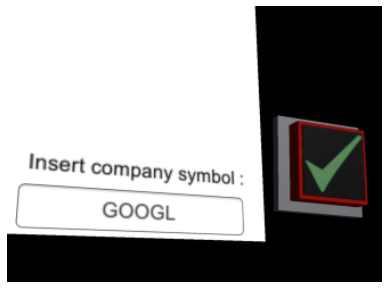

(c)

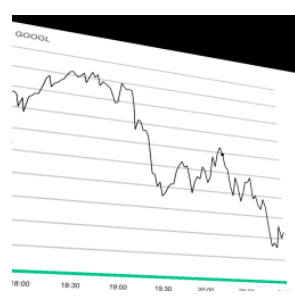

(d)

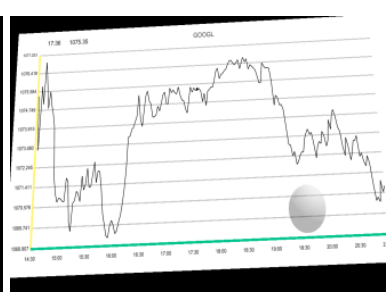

(e)

Figure 8

Creating and configuring virtual stock chart

A user can move a created chart anywhere around the user's space by pinching the red cube again. When the user is satisfied with the results and does not want to change anything, he or she can press the hammer button displayed in the upper right corner of the chart (Figure 8d). This switches the chart to the 'frozen' state, in which the red cube and the virtual keyboard disappear. Within this state, the possibility of using the keyboard and reconfiguring the chart is blocked. However, with the use of the grabbing gesture, a user can again grasp the virtual chart and move it to another position or adjust its orientation in space (Figure 8e). 
While creating financial TV channel screens, all activities are performed in the same way. A user goes through the same steps, except that a TV stream can be played and paused by pressing the center of the virtual screen component with a finger.

The system allows a user to preserve a created workspace. When a user is satisfied with the created virtual environment, he or she can save the environment. On the right side of the user, there are textual instructions with two buttons that can be used to save or load the virtual workspace (as depicted in Figure 9). The system saves identifiers of stock exchanges, codes of the shares, and coordinates of the created financial components within an external CSV file.

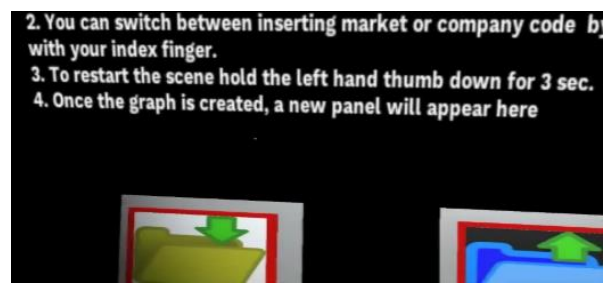

Figure 9

Displaying the interface responsible for saving and loading a virtual workspace

\section{Experimental Evaluation}

An experimental user evaluation of the presented system has been conducted. We used the Mode \#2 of system operation (tutorial) for practicing particular interactions required to build and configure an AR environment. In this experiment, we wanted to check how non-experienced users perform while creating and parameterizing a virtual chart within an AR environment with the use of hand gestures. We also intended to verify whether cognitive capabilities of users enable them to efficiently use interactive virtual screens in their surroundings. The following subsections cover the design of the experiment, the characteristics of participants that took part in the study, as well as the collected results and analysis.

\subsection{Design of the Experiment}

We designed three separated tasks to perform, as follows:

T1 - Pinching and moving red/blue cubes to swap their positions; aim: using the pinching gesture to interact with the UI and finding out whether users can sense the distance to virtual cubes. 
T2 - Writing the following text: "Badania.Naukowe" using the virtual keyboard; aim: using the punching gesture while writing on the virtual keyboard and verifying the precision of users while manipulating hands in space.

T3 - Grabbing and moving two defined charts to swap their positions; aim: using the grabbing gesture to interact with virtual charts and verifying whether users are able to sense the distance to AR charts.

Before performing a particular task, each user was instructed on the task. Completion time of each task has been measured. After finishing each task, the participants were asked to complete a questionnaire with Likert scale of 1 (very difficult) to 5 (very easy).

\subsection{Participants}

A total of 22 test subjects ( 7 female and 15 male) participated in the experiment. The subjects ranged in age from 20 to 25 years ( $M=20.52$ years, $S D=1.16$ years). None of the subjects had earlier experience with AR interface controlled with hand gestures.

\subsection{Results and Analysis}

Within this subsection, the results of the experiment are reported. The results include task completion times, questionnaire data gathered from the participants, as well as analysis of the data.

\subsubsection{Task Completion Times}

While conducting the experiment, each task completion time was measured for all the participants. The mean time taken to complete the task, as presented in Figures $10 \mathrm{a}, 10 \mathrm{~b}, 10 \mathrm{c}$, was analyzed using an independent t-test for two groups of users: female and male. The two-sample unequal variance (heteroscedastic) test was performed. The mean value of $\mathrm{T} 1$ for females equals $30.14 \mathrm{~s}$, while for males $33.67 \mathrm{~s}$. The female group was slightly faster, but the difference is no statistically significant. The same conclusions can be drawn for T3. However, in the case of $\mathrm{T} 2$, the results of an independent t-test with $95 \%$ confidence interval confirmed the existence of a significant difference between performance of male and female groups $\mathrm{p}=0.046$ ( $\mathrm{p}<0.05)$. The male group performed faster than female group. Females committed more typographical errors that had to be corrected with virtual backspace button than males. 


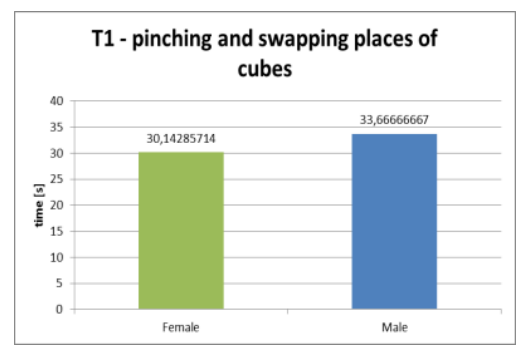

(a)

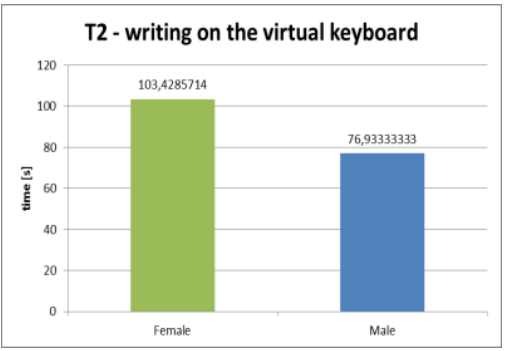

(b)

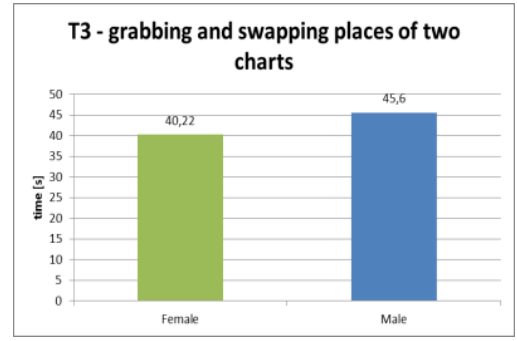

(c)

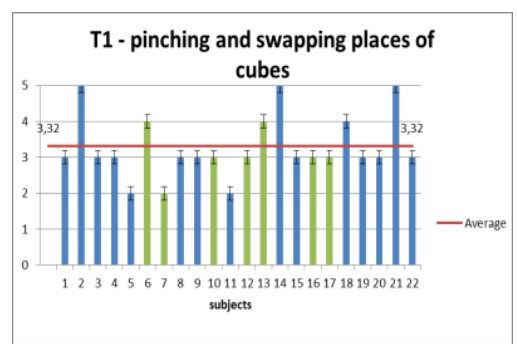

(d)

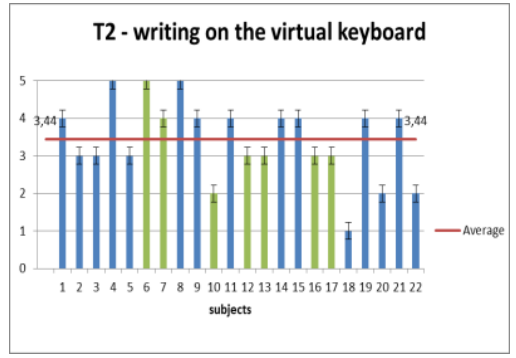

(e)

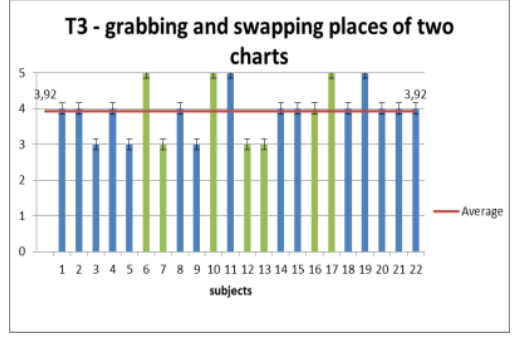

(f)

Figure 10

Collected results - (a), (b), (c): task completion times, (e), (d), (f): subjects' assessment

\subsubsection{Questionnaire: Likert Scale Rating}

Figures 10d, 10e, 10f summarize collected results of the assessment questionnaire. T1 was difficult only for 3 subjects ( 1 female, 2 males), T2 was very difficult only for 1 female and 2 males; also 1 male reported that typing on the virtual keyboard was a very difficult task to complete. T3 did not cause major difficulties for anyone (min. reported value $=3$ ). We observed that the grabbing gesture was natural for most participants. Only 6 subjects ( 3 females and 3 males) reported that the task was neither difficult nor easy. The results suggest that grabbing virtual charts was natural for the participants and they did not have major difficulties with this task. 
All participants completed all three tasks with success - even if a participant committed an error while writing on the virtual keyboard, he/she was able to correct the error and continue the work on the tasks. The results suggest that users who have had more problems with interacting with virtual objects needed more time to learn and get used to the AR interface enhanced with hand gestures. Such users had the biggest problem with distance perception and consequently with precision. They could not sense how far the cube is located and when they were writing on the virtual keyboard, they often pushed a wrong key or hit two keys at the same time. The AR environment required greater attention divisibility from users. Participants with the weakest results claimed that they could not focus on virtual objects; the real environment distracted them too much, comparing with the rest of users who did not report significant problems. However, users who scored the questionnaire with a value of 3 or higher were very satisfied. They could sense the distance between their hands and virtual objects. Moreover, they precisely performed tasks without significant difficulties compared with the users who did report problems. Moreover, subjects often expressed an opinion that the AR interface could give great opportunities for future work of equity traders.

\section{Conclusions and Future Work}

In this paper, we presented a novel AR Trading system that can be used by financial equity traders. The presented Augmented Reality system for visualization of financial stock data blends the cognition of artificial and natural elements in user's environment. CogInfoCom and augmented reality research expose strong synergies in the aspects of human-machine interaction, especially when performing IT related tasks [31] or collaborative learning in mixed reality environments [32].

The main objective of the presented study was to evaluate three fundamental tasks required to build and parametrize virtual stock charts within users' surroundings. Collected data suggest that users completed tasks without major difficulties. Only few reported problems while performing $\mathrm{T} 1$ and $\mathrm{T} 2$.

There are many areas of potential future research. In particular, we plan to perform an experimental study comparing user performance in VR versus AR mode. The nVisor device provides a removable cover that can be quickly applied to "close" the user in a VR environment, where the subject can see 3D models of hands to interact with the system. Another area that could be explored is to combine a real-world trading workspace with the AR interface and test how traders interact in such an environment. 


\section{Acknowledgments}

This research work has been supported by the Polish National Science Centre (NCN) Grant No. DEC-2016/20/T/ST6/00590.

\section{References}

[1] M. Billinghurst and $\mathrm{H}$. Kato. Collaborative augmented reality. Communications of the ACM, 45(7):64-70, 2002

[2] W. Cellary, W. Wiza, K. Walczak. Visualizing web search results in 3D, Computer 37(5), pp. 87-89, 2004

[3] Y. S. Chang, B. Nuernberger, B. Luan, and T. Höllerer. Evaluating gesturebased augmented reality annotation. In 2017 IEEE Symposium on 3D User Interfaces (3DUI), pp. 182-185, March 2017, doi: 10. 1109/3DUI.2017.7893337

[4] B. Fernandes and J. Fernandez. Bare hand interaction in tabletop augmented reality. In SIGGRAPH'09: Posters, p. 98, ACM, 2009

[5] K. Gupta, G. A. Lee, and M. Billinghurst. Do you see what I see? The effect of gaze tracking on task space remote collaboration. IEEE transactions on visualization and computer graphics, 22(11):2413-2422, 2016

[6] G. Heidemann, I. Bax, and H. Bekel. Multimodal interaction in an augmented reality scenario. In Proceedings of the $6^{\text {th }}$ international conference on Multimodal interfaces, pp. 53-60, ACM, 2004

[7] Khan, Humayun, et al. "Evaluating the Effects of Hand-gesture-based Interaction with Virtual Content in a $360^{\circ}$ Movie." (2017)

[8] E. Kaiser, A. Olwal, D. McGee, H. Benko, A. Corradini, X. Li, P. Cohen, and S. Feiner. Mutual disambiguation of 3D multimodal interaction in augmented and virtual reality. In Proceedings of the $5^{\text {th }}$ international conference on Multimodal interfaces, pp. 12-19, ACM, 2003

[9] M. Kolsch, R. Bane, T. Hollerer, and M. Turk. Multimodal interaction with a wearable augmented reality system. IEEE Computer Graphics and Applications, 26(3):62-71, 2006

[10] T. Piumsomboon, D. Altimira, H. Kim, A. Clark, G. Lee, and M. Billinghurst. User-defined gestures for augmented reality. In CHI'13 Extended Abstracts on Human Factors in Computing Systems, pp. 955-960, ACM, 2013

[11] T. Piumsomboon, D. Altimira, H. Kim, A. Clark, G. Lee, and M. Billinghurst. Grasp-shell vs gesture-speech: A comparison of direct and indirect natural interaction techniques in augmented reality. In 2014 IEEE International Symposium on Mixed and Augmented Reality (ISMAR), pp. 73-82, Sept 2014. doi: 10.1109/ISMAR.2014. 6948411 
[12] D. Ruminski. An experimental study of spatial sound usefulness in searching and navigating through AR environments. Virtual Reality, 19(3):223-233, Nov 2015. doi: 10.1007/s10055-015-0274-4

[13] J. Sodnik, S. Tomazic, R. Grasset, A. Duenser, and M. Billinghurst. Spatial sound localization in an augmented reality environment. In Proceedings of the 18th Australia Conference on Computer-Human Interaction: Design: Activities, Artefacts and Environments, OZCHI '06, pp. 111-118, ACM, New York, NY, USA, 2006. doi: 10.1145/ 1228175.1228197

[14] T. Taehee Lee and A. Handy. Markerless inspection of augmented reality objects using fingertip tracking. In IEEE International Symposium on Wearable Computers, 2007

[15] P. P. Valentini and E. Pezzuti. Accuracy in fingertip tracking using leap motion controller for interactive virtual applications. International Journal on Interactive Design and Manufacturing (IJIDeM), 11(3):641-650, 2017

[16] K. Walczak, W. Cellary. IEEE Symposium on Applications and the Internet (SAINT 2002) Nara, Japan, pp. 204-211, IEEE Computer Society, 2002

[17] R. Wang, S. Paris, and J. Popovic. 6d hands: markerless hand-tracking ' for computer aided design. In Proceedings of the $24^{\text {th }}$ annual ACM symposium on User interface software and technology, pp. 549-558, ACM, 2011

[18] C.-M. Wu, C.-W. Hsu, T.-K. Lee, and S. Smith. A virtual reality keyboard with realistic haptic feedback in a fully immersive virtual environment. Virtual Reality, 21(1):19-29, Mar 2017, doi: 10.1007/ s10055-016-0296-6

[19] Z. Zhou, A. D. Cheok, X. Yang, and Y. Qiu. An experimental study on the role of software synthesized 3D sound in augmented reality environments. Interacting with Computers, 16(5):989-1016, 2004

[20] A. Zocco, M. D. Zocco, A. Greco, S. Livatino, and L. T. De Paolis. Touchless Interaction for Command and Control in Military Operations, pp. 432-445, Springer International Publishing, Cham, 2015. doi: 10. 1007/978-3-319-22888-4 32

[21] Jacques Cohen. Communications of the ACM - Special issue on computer augmented environments: back to the real world, 36(7), 1993

[22] Ivan E. Sutherland. A head-mounted three dimensional display. In Proceedings of the December 9-11, 1968, Fall Joint Computer Conference, Part I, AFIPS '68 (Fall, part I), pp. 757-764, New York, NY, USA, 1968, ACM

[23] Paul Milgram, Haruo Takemura, Akira Utsumi, and Fumio Kishino. Augmented reality: a class of displays on the reality-virtuality continuum. Vol. 2351, pages 282-292, 1995

[24] Ronald T Azuma. A survey of augmented reality. Presence, 6(4):355-385, 1997 
[25] P. Baranyi and Á. Csapó. Cognitive infocommunications: Coginfocom. $11^{\text {th }}$ International Symposium on Computational Intelligence and Informatics (CINTI), pp. 141-146, 2010

[26] P. Baranyi and Á. Csapó. "Definition and synergies of cognitive infocommunications." Acta Polytechnica Hungarica 9.1, pp. 67-83, 2012

[27] Á. Csapó and P. Baranyi. A unified terminology for the structure and semantics of CogInfoCom channels. Acta Polytechnica Hungarica, 9(1), pp. $85-105,2012$

[28] P. Baranyi, Á. Csapó and P. Varlaki. An overview of research trends in CogInfoCom. IEEE $18^{\text {th }}$ International Conference on Intelligent Engineering Systems INES 2014, Tihany, pp. 181-186, 2014

[29] P. Baranyi, Á. Csapó and G. Sallai. Cognitive Infocommunications (CogInfoCom) Springer, 2015

[30] A. Pongrácz and J. Sipos, Teaching coaching using 3D/VR technology in the light of intercultural knowledge, $20178^{\text {th }}$ IEEE International Conference on Cognitive Infocommunications (CogInfoCom) Debrecen, pp. 000530-000530, 2017

[31] G. Sziladi, T. Ujbanyi, J. Katona and A. Kovari, The analysis of hand gesture based cursor position control during solve an IT related task, 2017 $8^{\text {th }}$ IEEE International Conference on Cognitive Infocommunications (CogInfoCom), Debrecen, pp. 000413-000418, 2017

[32] V. Kövecses-Gösi, (2018). Cooperative Learning in VR Environment. Acta Polytechnica Hungarica, 15(3) 